\title{
Anhang Physikalische Konstanten
}

Gravitationskonstante $\quad G=6,67 \cdot 10^{-11} \frac{\mathrm{Nm}^{2}}{\mathrm{~kg}^{2}}$,

Lichtgeschwindigkeit $\quad c=299.792 .458 \frac{\mathrm{m}}{\mathrm{s}} \approx 3 \cdot 10^{8} \frac{\mathrm{m}}{\mathrm{s}}$,

Boltzmann-Konstante $\quad k_{B}=1,38 \cdot 10^{-23} \frac{\mathrm{J}}{\mathrm{K}}$,

Avogadro Konstante $\quad N_{A}=\frac{6,02 \cdot 10^{23}}{\mathrm{~mol}}$,

Allgemeine Gaskonstante $\quad R=8,31 \frac{\mathrm{J}}{\mathrm{mol} \cdot \mathrm{K}}$,

Elektrische Feldkonstante $\quad \varepsilon_{0}=8,85 \cdot 10^{-12} \frac{\mathrm{As}}{\mathrm{Vm}}$,

Elementarladung $\quad e=1,6 \cdot 10^{-19} \mathrm{C}$,

Magnetische Feldkonstante $\quad \mu_{0}=4 \pi \cdot 10^{-7} \frac{\mathrm{Tm}}{\mathrm{A}}$,

Stefan-Boltzmann Konstante $\quad \sigma=5,67 \cdot 10^{-8} \frac{\mathrm{W}}{\mathrm{m}^{2} \cdot \mathrm{K}^{4}}$,

Planck'sches Wirkungsquantum $\quad h=2 \pi \cdot \hbar=6,63 \cdot 10^{-34} \mathrm{Js}$,

Bohrradius $a_{B}=0,5 \cdot 10^{-10} \mathrm{~m}$,

Rydberg-Energie $\quad E_{\mathrm{Ry}}=13,6 \mathrm{eV}$,

Masse des Elektrons $\quad m_{e}=9,1 \cdot 10^{-31} \mathrm{~kg}$,

Masse des Protons $\quad m_{p}=1,673 \cdot 10^{-27} \mathrm{~kg}$,

Masse des Neutrons $\quad m_{n}=1,675 \cdot 10^{-27} \mathrm{~kg}$,

Atomare Masseneinheit $u=1,661 \cdot 10^{-27} \mathrm{~kg}$. 